\title{
Nonlocal Symmetry Breaking in Kaluza-Klein Theories
}

\author{
A. Masiero and M. Serone \\ ISAS-SISSA, Via Beirut 2-4, I-34013 Trieste and INFN, sezione di Trieste, Italy \\ C. A. Scrucca \\ CERN, 1211 Geneva 23 Switzerland \\ L. Silvestrini \\ INFN, sezione di Roma, Dipartimento di Fisica, Università di Roma "La Sapienza," \\ Piazzale Aldo Moro 2, I-00185, Roma, Italy \\ (Received 27 July 2001; published 3 December 2001)
}

\begin{abstract}
Scherk-Schwarz gauge symmetry breaking of a $D$-dimensional field theory model compactified on a circle is analyzed. It is explicitly shown that forbidden couplings in the unbroken theory appear in the one-loop effective action only in a nonlocal way, implying that they are finite at all orders in perturbation theory. This result can be understood as a consequence of the local gauge symmetry, but it holds true also in the global limit.
\end{abstract}

DOI: $10.1103 /$ PhysRevLett.87.251601

There has been recently a vast amount of work exploring the implications of breaking gauge [1] or supersymmetries [2-4] via the Scherk-Schwarz (SS) mechanism [5]. Indeed, this mechanism, which until recently had not been put into action in explicit theories, has revealed exciting, but also puzzling aspects. In particular, amazing ultraviolet softness properties in theories with extra dimensions where supersymmetry (SUSY) breaking occurs through the SS mechanism have sparked a vast debate on what is at the root of such "finiteness protection" (a protection which is even more efficient than that provided by softly broken supersymmetry in ordinary four-dimensional theories).

The physical observables which have first drawn attention on this apparently surprising ultraviolet behavior of extra-dimensional supersymmetric theories are the scalar masses [3]. Although the higher-dimensional theory is generally nonrenormalizable, it was pointed out that the radiative corrections to the scalar masses are quite insensitive to the cutoff $\Lambda$ if the compact space is of size $O\left(\mathrm{TeV}^{-1}\right)$. However, the rationale for such finiteness is still debatable [6]. At a more technical level, doubts were cast on the physical meaning of the commonly adopted "Kaluza-Klein (KK) regularization" consisting in summing over the infinite tower of KK states and performing the one-loop integral over the momenta. It was argued that the quadratic dependence of the Higgs masses on the ultraviolet cutoff could reappear in higher orders in perturbation theory given the ad hoc character of the KK regularization.

This Letter aims at clarifying the debate by analyzing the SS breaking mechanism in a simpler gauge model in $D$ dimensions compactified on a circle down to $D-1$ dimensions with arbitrary boundary conditions. We compute one-loop corrections to scalar masses and explicitly show that the SS breaking manifests itself only via the appearance of terms which are nonlocal along the compact dimension. Although we cannot provide an equivalent de-
PACS numbers: 11.10.Kk, 11.15.Ex, 11.25.Mj, 11.30.Qc

scription for orbifolds and for the case in which the SS mechanism, together with projections, is invoked to break SUSY, we think that the main idea is basically the same, namely, the Higgs mass correction is finite because it is necessarily a nonlocal term in the one-loop effective action [7].

Here are our main results. First we observe that to get a sensible result the sum over the whole tower of KK states has to be performed, since any acceptable regularization has to respect the local symmetries in $D$ dimensions which are not actually broken when the SS mechanism takes place. The Ward identities can be recovered as in the unbroken case, but with twisted gauge parameters. Alternatively, following [9], the SS mechanism can be viewed as a spontaneous breaking through the vacuum expectation value (VEV) of $A_{D}$, namely, the component of the connection along the compact coordinate. Any truncation of the sum would correspond to a regularization which does not respect the symmetries of the theory. Second and more important of our results is that the nonlocal nature of the SS breaking appears in the Lagrangian through nonlocal terms. More precisely, terms that would be forbidden in the unbroken theory may now appear, but only as nonlocal ones, associated with Wilson lines and Wilson loops. Hence, for such terms, the breaking à la SS corresponds to a nonlocal spontaneous symmetry breaking. The nonlocal symmetric terms in the $D$-dimensional Lagrangian correspond to the nonsymmetric local terms of the spontaneously broken theory in $D-1$ dimensions. As an illustration of this result, we analyze the mass splitting in a $\mathrm{SU}(2) \times \mathrm{U}(1)$ scalar doublet and its generalization for a $\mathrm{SU}(N)$ model.

The nonlocality of these gauge-invariant terms arising from the SS breaking is at the root of the finiteness of the radiative corrections we compute. In a renormalized quantum field theory, at any order in perturbation theory, 
all divergent terms are polynomial in the external momenta. Therefore, they correspond to local counterterms to be added to the Lagrangian. Since the only symmetrybreaking quantities generated by radiative corrections in this model correspond to nonlocal gauge-invariant terms, they cannot be divergent. This argument can also be applied to nonrenormalizable field theories. Hence we can conclude that the finiteness of radiatively induced symmetry-breaking terms has to persist at all orders in perturbation theory. An important comment is however in order. Our results show that any symmetry-breaking term, being necessarily nonlocal, is finite, but this does not imply at all that the physical values of the associated quantities are finite and cutoff independent. In the case we study, of course, divergences appear in local gaugeinvariant terms. Being that the underlying theory is nonrenormalizable (for $D>4$ ), a certain sensitivity on the cutoff is therefore present and unavoidable, but it is confined to local gauge-invariant quantities.

Let us then consider the following $D$-dimensional $\mathrm{SU}(2) \times \mathrm{U}(1)$ gauge theory [the reason of the additional $\mathrm{U}(1)$ factor will be clear later on]:

$$
\begin{aligned}
\mathcal{L}= & \left(D_{M} \Phi\right)^{\dagger}\left(D^{M} \Phi\right)+\bar{\Psi} i \not \supset \Psi+\bar{\chi} i \not \partial \chi-\frac{1}{8 g^{2}} \operatorname{Tr} F_{M N}^{2} \\
& -\frac{1}{4 g^{\prime 2}} F_{M N}^{\prime 2}+\lambda\left(\Phi^{\dagger} \bar{\chi} \Psi+\text { H.c. }\right)
\end{aligned}
$$

where $\Phi$ and $\Psi$ are U(1)-charged SU(2) doublets and $\chi$ is a singlet, $D_{M}=\partial_{M}-i A_{M}^{a} T^{a}-i A^{0}$, with $T^{a}$ the Pauli matrices and $A^{0}$ the $\mathrm{U}(1)$ connection, $M=(\mu, D), \mu=$ $0, \ldots, D-2$ and $\not D=D_{M} \gamma^{M}$.

We take the $D$ th dimension to be a circle of radius $R$. The SS breaking is achieved by imposing the following periodicities on the fields in Eq. (1):

$$
\begin{aligned}
\Phi(y+2 \pi R) & =U \Phi(y) \quad \Psi(y+2 \pi R)=U \Psi(y), \\
A_{M}(y+2 \pi R) & =U A_{M}(y) U^{\dagger} \quad \chi(y+2 \pi R)=\chi(y),
\end{aligned}
$$

where $y$ denotes the compact coordinate and

$$
U \equiv U(\alpha, \beta)=e^{2 i \pi\left(\alpha T_{3}+\beta\right)} .
$$

The corresponding Fourier decomposition of a generic field $\phi$ along the compact dimension is given by

$$
\phi(x, y)=\frac{1}{\sqrt{2 \pi R}} \sum_{n=-\infty}^{+\infty} e^{i(n+a) y / R} \phi_{n}(x),
$$

where $x$ represents the $(D-1)$-dimensional coordinates. The values of $a$ for the above fields are as follows: $A^{0,3}$ and $\chi$ remain periodic $(a=0), a=\alpha+\beta$ for the upper components $\phi_{1}$ and $\psi_{1}$ of the doublets, $a=-\alpha+\beta$ for the lower components $\phi_{2}$ and $\psi_{2}$, and $a= \pm 2 \alpha$ for $A^{ \pm} \equiv\left(A^{1} \pm i A^{2}\right) / \sqrt{2}$. These periodicities are conserved by any single-valued gauge transformation on the circle.

It is interesting to note that if the gauge parameters $\varepsilon_{a}(x, y)$ have the same periodicity as the corresponding gauge fields in Eq. (2), the compactified theory is still invariant under the full $\mathrm{SU}(2) \times \mathrm{U}(1)$ gauge group. Of course, since $\varepsilon_{1,2}(x, y)$ do not admit the rigid limit in $y$, from the $(D-1)$-dimensional point of view the unbroken gauge group is only $\mathrm{U}(1) \times \mathrm{U}(1)$. Furthermore, as shown by Hosotani [9], one can perform more general gauge transformations, changing the boundary conditions for the fields in Eq. (2). In particular, it is possible to obtain the case in which all fields are periodic, by means of the following gauge transformation:

$$
\Omega(y)=e^{-i\left(\alpha T_{3}+\beta\right) y / R} .
$$

However, the $D$ th component of the transformed gauge fields acquires a vacuum expectation value given by

$$
\left\langle A_{D}\right\rangle=-\left(\alpha T_{3}+\beta\right) / R \text {. }
$$

Indeed, one can check that this VEV reproduces the mass spectrum obtained from Eq. (2). Therefore, the SS breaking of a gauge symmetry can be alternatively seen, when all fields are periodic, as a spontaneous symmetry breaking induced by the VEV of $A_{D}$.

Since the gauge symmetry is broken in a peculiar way through the boundary conditions or, alternatively, through a VEV for $A_{D}$ [10], we expect that SU(2)-breaking terms should be nonlocal around the compact coordinate. It is our aim to explicitly show that this is indeed what happens, by considering mass terms induced in the one-loop effective action for the $\Phi$ doublet. We compute the two-point functions for on-shell external momenta, $q^{2}=q_{D}^{2}$, in order to extract directly the renormalized mass terms.

To better discuss the global limit $g, g^{\prime} \rightarrow 0$ of our results, we first compute the fermionic contribution to the two-point function, which we denote by $\mathcal{F}_{a}\left(q^{2}, q_{D}\right)$, where $a=\beta \pm \alpha$ for the doublet. This is given by

$$
i \mathcal{F}_{a}\left(q^{2}, q_{D}\right)=\frac{\lambda^{2} 2^{[D / 2]}}{2 \pi R} \int \frac{d^{D-1} p}{(2 \pi)^{D-1}} \sum_{p_{D}} \frac{p \cdot(p+q)-p_{D}\left(p_{D}+q_{D}\right)}{\left(p^{2}-p_{D}^{2}\right)\left[(p+q)^{2}-\left(p_{D}+q_{D}\right)^{2}\right]},
$$

where $[D / 2]=\operatorname{int}(D / 2)$ takes into account the dimensionality of the gamma matrices in $D$ dimensions. The sum over the KK tower can be easily performed and one finds, going to the Euclidean, at $q^{2}=q_{D}^{2}$,

$$
\begin{aligned}
\mathcal{F}_{a}\left(q_{D}^{2}, q_{D}\right)= & -\frac{\lambda^{2} 2^{[D / 2]-1} R^{2-D}}{(4 \pi)^{(D-1) / 2} \Gamma\left(\frac{D-1}{2}\right)} \operatorname{Re} \int_{0}^{\infty} d x \\
& \times x^{D-3}[\operatorname{coth} \pi x+\operatorname{coth} \pi(x+i a)],
\end{aligned}
$$

where $x=p R$. Interestingly, the result does not depend on the KK level $q_{D}=(m+a) / R$ and, hence, each KK mode $\Phi_{m}$ of the doublet gets a universal one-loop correction to its mass. This property is a consequence of the $D$-dimensional local gauge invariance that is still present in the theory and would have been rather obscure from a purely $(D-1)$-dimensional point of view. 
It is convenient to define $\mathcal{F}_{ \pm}=\mathcal{F}_{\beta+\alpha}\left(q_{D}^{2}, q_{D}\right) \pm$ $\mathcal{F}_{\beta-\alpha}\left(q_{D}^{2}, q_{D}\right)$, encoding, respectively, the $\mathrm{SU}(2)$ symmetric and $\mathrm{SU}(2)$-breaking parts. The momentum integral can be explicitly evaluated and one obtains

$$
\begin{aligned}
\mathcal{F}_{+}= & \lambda^{2} 2^{[D / 2]+1} \\
& \times\left[\Lambda^{D-2}+\sum_{n=1}^{\infty} C_{D}^{(n)} \cos 2 \pi n \alpha \cos 2 \pi n \beta\right], \\
\mathcal{F}_{-}= & -\lambda^{2} 2^{[D / 2]+1} \sum_{n=1}^{\infty} C_{D}^{(n)} \sin 2 \pi n \alpha \sin 2 \pi n \beta,
\end{aligned}
$$

where $\quad C_{D}^{(n)}=(D-3) ! /\left[(4 \pi)^{(D-1) / 2} \Gamma\left(\frac{D-1}{2}\right)(2 \pi \times\right.$ $\left.R n)^{D-2}\right]$. The $(\alpha, \beta)$-independent term in (9) represents the usual divergence that one gets in the unbroken case. Since gauge invariance does not forbid the appearance of such a term, it is generated with the expected degree of divergence.

The form of the one-loop induced $\mathrm{SU}(2)$-breaking mass term for $\Phi$ can now be derived by taking the Fourier transform of (9). It yields the following nonlocal coupling:

$$
\begin{aligned}
\mathcal{L}_{\mathrm{nl}}^{\mathcal{F}}= & \lambda^{2} 2^{[D / 2]-1} \sum_{n=1}^{\infty} C_{D}^{(n)} \Phi^{\dagger}(y) W_{n} \Phi(y+2 \pi R n) \\
& + \text { H.c. },
\end{aligned}
$$

involving Wilson lines around the compact direction:

$$
W_{n}=W_{n}(y)=\mathcal{P} e^{i \int_{y}^{y+2 \pi R n} A_{D}\left(y^{\prime}\right) d y^{\prime}} .
$$

In the picture in which $\left\langle A_{D}\right\rangle=-\left(\alpha T_{3}+\beta\right) / R$ and the boundary conditions are periodic, one finds

$\operatorname{Re}\left\langle W_{n}\right\rangle=\cos 2 \pi n \alpha \cos 2 \pi n \beta-T_{3} \sin 2 \pi n \alpha \sin 2 \pi n \beta$,

reproducing the correct $(\alpha, \beta)$ dependence found in (9). Alternatively, in the picture in which $\left\langle A_{D}\right\rangle=0$ but the boundary conditions are twisted, one has

$$
\Phi(y+2 \pi R n)=U^{n}(\alpha, \beta) \Phi(y),
$$

and the same result is obtained, as expected. Equation (10) shows explicitly that $\mathrm{SU}(2)$-breaking terms must be nonlocal around the compact direction, and hence finite.

Let us now consider the gauge contribution to the twopoint function, denoted by $G_{a}\left(q^{2}, q_{D}\right)$. The general formula can be written as

$$
i G_{a}\left(q^{2}, q_{D}\right)=\frac{1}{2 \pi R} \int \frac{d^{D-1} p}{(2 \pi)^{D-1}} \sum_{\text {colors }} \sum_{p_{D}}\left[\frac{-2 D}{p^{2}-p_{D}^{2}}+\frac{(p+2 q)^{2}-\left(p_{D}+2 q_{D}\right)^{2}}{\left(p^{2}-p_{D}^{2}\right)\left[(p+q)^{2}-\left(p_{D}+q_{D}\right)^{2}\right]}\right],
$$

omitting the group generators and couplings and the $p_{D}$ dependence on the colors. Defining symmetric and breaking parts $G_{ \pm}$as before and fixing $q^{2}=q_{D}^{2}$, one finds for $\mathrm{SU}(2) \times \mathrm{U}(1)$

$$
\begin{aligned}
\mathcal{G}_{+}= & -4 \sum_{n=1}^{\infty} C_{D}^{(n)}\left[\left(3 g^{2}+g^{\prime 2}\right) \cos 2 \pi n \alpha \cos 2 \pi n \beta\right. \\
& \left.+4 g^{2}(D-1) \cos 4 \pi n \alpha\right], \\
\mathcal{G}_{-}= & 4\left(g^{\prime 2}-g^{2}\right) \sum_{n=1}^{\infty} C_{D}^{(n)} \sin 2 \pi n \alpha \sin 2 \pi n \beta,
\end{aligned}
$$

where we omitted a $(\alpha, \beta)$ independent divergence in $\mathcal{G}_{+}$. Notice that $G_{ \pm}$do not have precisely to coincide with those of Eq. (9), because they mix up with Wilson loop contributions. In fact, these represent clearly another class of terms that are finite because they are nonlocal around the compact dimension (see also [11]). New nonlocal structures are therefore present. In particular, the effective coupling gets also Wilson loop contributions:

$$
\begin{aligned}
\mathcal{L}_{\mathrm{nl}}^{G}= & -\sum_{n=1}^{\infty} C_{D}^{(n)}\left\{\left[g^{\prime 2}-g^{2}+4 g^{2}(D-1) \operatorname{Tr} W_{n}^{\dagger}\right]\right. \\
& \times \Phi^{\dagger}(y) W_{n} \Phi(y+2 \pi R n) \\
& \left.+2 g^{2} \operatorname{Tr} W_{n} \Phi^{\dagger}(y) \Phi(y)\right\}+ \text { H.c. }
\end{aligned}
$$

Notice that for $g=g^{\prime}$ [the U(2) case], $\mathcal{G}_{-}$vanishes and for the pure $\mathrm{SU}(2)$ case, i.e. $\beta=0$, both $\mathcal{G}_{-}$and $\mathcal{F}_{-}$vanish (this is a general feature of $\mathrm{SU}(2 M)$ groups; see below). No SU(2) breaking terms are generated in this case.
Although our analysis was restricted to the one-loop approximation, it is clear that the nonlocality of symmetrybreaking couplings will persist at any order of perturbation theory, never allowing for the appearance of UV divergences. These occur in configuration space at very short distances, but all the effective symmetry-breaking interactions involve Wilson lines winding around the compact direction and can never produce short-distance singularities. Indeed, we argued that radiative corrections preserve the symmetries of the $D$-dimensional theory and that the Wilson line is the only quantity that can be generated leading to symmetry-breaking terms from the $(D-$ 1)-dimensional point of view. Thus, any higher order nonsymmetric contribution will be a function of the Wilson line, and it will be finite once all the relevant symmetric counterterms of lower order are added to all subgraphs.

Let us now consider the global limit $g, g^{\prime} \rightarrow 0$. In this case, the gauge field is clearly nondynamical, but its VEV (6), which is $g, g^{\prime}$ independent and still induces a nontrivial value of $W_{n}$, can be considered as a sort of leftover flux or condensate, responsible for the twist in the boundary conditions of the fields. The gauge contribution (15) now clearly vanishes and Eq. (10) reduces to

$$
\mathcal{L}_{\mathrm{nl}}=\lambda^{2} 2^{[D / 2]-1} \sum_{n=1}^{\infty} C_{D}^{(n)} \Phi^{\dagger}(y) \Phi(y+2 \pi R n)+\text { H.c. },
$$

with the periodicities as in (12). It is interesting to notice 
that the terms in (16) can be alternatively considered as nonlocal and (global) SU(2) invariant, written as above, or local and SU(2) breaking using (12). It is clear, however, that finiteness is also in this case ensured at all orders in perturbation theory.

An alternative approach to the one followed here consists in computing the two-point functions at $q=0$ and generic $q_{D}=(m+a) / R$. In this way, one gets an explicit $(m+a)$ dependence in the amplitudes that, when the latter is reinterpreted as $-i \partial_{D}$, leads to an expansion in derivatives for an infinite number of terms. We checked

$$
\mathcal{L}_{\mathrm{nl}}^{\mathrm{SU}(N)}=-g^{2} \sum_{n=1}^{\infty} C_{D}^{(n)}\left\{\left[-\frac{2}{N}+4(D-1) \operatorname{Tr} W_{n}^{\dagger}\right] \Phi^{\dagger}(y) W_{n} \Phi(y+2 \pi R n)+2 \operatorname{Tr} W_{n} \Phi^{\dagger}(y) \Phi(y)\right\}+\text { H.c. }
$$

For $N=2 M$, one has $a=b=M$ so that $\operatorname{Re} W_{n}$ is an even function of $\alpha$ and $\operatorname{Tr} W_{n}$ is real. It follows that no mass splitting between the upper and lower components of the $2 M$ plet is radiatively generated. On the contrary, for $N=2 M+1$, one has $a=M, b=M+1$ and a mass splitting between the upper $M+1$ and lower $M$ components is produced at one loop.

Similarly, one can also study the one-loop amplitude $\left\langle A_{D} A_{D}\right\rangle$. For example, in the $\mathrm{SU}(2) \times \mathrm{U}(1)$ model, one finds that all KK levels but the zero modes of $A_{D}^{0}$ and $A_{D}^{3}$ do not receive any correction, whereas the latter get a finite and $(\alpha, \beta)$-dependent correction. This is what is expected. Gauge symmetry forbids the appearance of a mass term for $A_{M}$, whereas a one-loop effective potential for $A_{D, 0}^{0,3}$ is generated. These zero modes are the nonintegrable phases of [9], related to the eigenvalues of the Wilson loop.

Our results hold by summing over the entire tower of KK states. One can easily verify that new and $(\alpha, \beta)$ dependent divergences appear (for $D>4$ ), also in symmetry-breaking terms, as soon as the sum over KK modes is truncated. This is due to the fact that the local gauge symmetry along the compact coordinate is explicitly broken in this way.

As we have already mentioned, we did not consider the case in which the SS mechanism is implemented through $R$-symmetry transformations to break SUSY and induce a finite Higgs mass [4]. An explicit computation in this context, along the lines considered here, would be very interesting. An important and related open issue is to understand whether the SS mechanism applied to an $R$ symmetry can be viewed as a spontaneous breaking of local supersymmetry, very much along the lines of [9]. Recent work suggests that this might actually be the case [12], but a full supergravity analysis is required to definitively establish this result.

We thank N. Arkani-Hamed, R. Barbieri, A. Donini, P. Gambino, H. Gies, L. Griguolo, G. Isidori, and F. Zwirner for interesting discussions. This work has been partially supported by the EEC through the RTN network that again all the $\mathrm{SU}(2)$ breaking terms are finite. Moreover, as expected, the leading $q_{D}=0$ mass term coincides with (9) and (14).

The analysis that we have performed for the $\mathrm{SU}(2) \times$ $\mathrm{U}(1)$ model can be easily extended to a general $\mathrm{SU}(N)$ model with (3) given by $U=\exp (2 i \pi \alpha T)$, where $T=$ $\operatorname{diag}\left(t_{1}, \ldots, t_{N}\right)$ and $t_{1, \ldots, b}=a / c, t_{(b+1), \ldots, N}=-b / c, c=$ $\sqrt{\left(b a^{2}+a b^{2}\right) / 2}$. The fermionic contribution Eq. (8) and the discussion following it remain valid, with $a$ in Eq. (8) being now the twist in $q_{D}$ of each component of the $N$ plet. For the gauge contribution, one finds instead the following effective coupling for the $\alpha$-dependent part:

"Across the Energy Frontier," Contract No. HPRN-CT2000-00122.

[1] Y. Kawamura, Prog. Theor. Phys. 103, 613 (2000); hep-ph/0012125; Prog. Theor. Phys. 105, 691 (2001); G. Altarelli and F. Feruglio, Phys. Lett. B 511, 257 (2001); L. Hall and Y. Nomura, hep-ph/0103125; A. Hebecker and J. March-Russel, hep-ph/0106166; hep-ph/0107039; R. Barbieri, L. J. Hall, and Y. Nomura, hep-ph/0106190.

[2] I. Antoniadis, Phys. Lett. B 246, 377 (1990).

[3] I. Antoniadis, C. Munoz, and M. Quiros, Nucl. Phys. B397, 515 (1993); I. Antoniadis, S. Dimopoulos, A. Pomarol, and M. Quiros, Nucl. Phys. B544, 503 (1999); A. Delgado, A. Pomarol, and M. Quiros, Phys. Rev. D 60, 095008 (1999).

[4] R. Barbieri, L. J. Hall, and Y. Nomura, Phys. Rev. D 63, 105007 (2001).

[5] J. Scherk and J. H. Schwarz, Nucl. Phys. B153, 61 (1979); Phys. Lett. 82B, 60 (1979).

[6] D. M. Ghilencea and H. Nilles, hep-ph/0103151; A. Delgado, G. von Gersdorff, P. John, and M. Quiros, hep-ph/0104112; R. Contino and L. Pilo, hep-ph/0104130; Y. Nomura, hep-ph/0105113; N. Weiner, hep-ph/0106021; H. D. Kim, hep-ph/0106072; R. Barbieri, L. J. Hall, and Y. Nomura, hep-th/0107004.

[7] The nonlocality of the SS breaking mechanism was already pointed out in [8], but its implications for the effective Lagrangian were not fully exploited.

[8] N. Arkani-Hamed, L. Hall, Y. Nomura, D. Smith, and N. Weiner, Nucl. Phys. B605, 81 (2001).

[9] Y. Hosotani, Phys. Lett. 126B, 309 (1983); 129B, 193 (1983); Ann. Phys. (N.Y.) 190, 233 (1989).

[10] Notice that also this last picture makes sense due to the presence of a compactified coordinate. In absence of the latter, constant gauge connections can always be gauged away.

[11] N. Arkani-Hamed, A. G. Cohen, and H. Georgi, hep-ph/ 0105239.

[12] D. Marti and A. Pomarol, hep-ph/0106265. 